\title{
PRIMER CASO DE THELAZIOSIS OCULAR (THELAZIA RHODESI) EN TORO DE LIDIA EN LA PENÍNSULA IBÉRICA
}

First case of ocular thelaziosis (Thelazía rhodesi) in fighting bull at the Iberian Peninsula

\author{
Seva, J. ${ }^{*}$; Sanes, J.M.1.; Mas, A.1; Bueno, J.M.³ Martínez-Carrasco, C².
}

${ }^{1}$ Departamento de Anatomía y Anatomía Patológica Comparadas. ${ }^{2}$ Departamento de Sanidad Animal. Facultad de Veterinaria. Campus de Excelencia Internacional Regional "Campus Mare Nostrum". Universidad de Murcia. Campus de Espinardo. Murcia, Spain. ${ }^{3}$ Laboratorio de Óptica. Centro de Investigación en Óptica y nanofísica. Universidad de Murcia. Campus de Espinardo. Murcia, Spain.

* Autor para correspondencia: Seva, Juan. Tel: +34 868884258. E-mail: jseva@um.es

Historial del artículo:

Enviado: 6 de junio, 2019

Aceptado:6 de marzo, 2021

\section{RESUMEN}

La thelaziosis bovina está causada por Thelazia rhodesi, T. skrjabini y T. gulosa, aunque la especie más prevalente en Europa es la primera de ellas. Thelazia rhodesi es un nematodo (orden Spirurida, familia Thelaziidae) que parasita el globo ocular del ganado vacuno, búfalos, cebúes, bisontes y, con menor frecuencia, caballos y pequeños rumiantes. Se localiza en la superficie corneal, sobre la membrana nictitante y en el saco conjuntival. Tiene un ciclo biológico indirecto, actuando como hospedadores intermediarios dípteros de la familia Muscidae. En este estudio se describe un caso de thelaziosis ocular en un toro de lidia (Bos taurus), muerto en la plaza de toros de Murcia en septiembre de 2014, procedente de una ganadería localizada en el suroeste de la Península Ibérica. Durante el primer y segundo reconocimiento veterinario del animal en la plaza de toros, se observó la existencia de lagrimeo abundante, pero a pesar de ello el animal reaccionaba positivamente a estímulos visuales realizados en el campo visual de ambos ojos, conjunta e independientemente. A pesar del lagrimeo constante, el animal fue considerado apto para la lidia. Durante esta no se observó anormalidad alguna en el comportamiento del toro en relación con el sentido de la vista. La inspección ocular post mortem realizada en el desolladero de la plaza permitió detectar la presencia de nematodos de 8-20 mm de longitud tanto en el 
saco conjuntival como en la superficie de la córnea, que fueron extraídos con ayuda de pinzas y conservados en etanol al 70\%. La identificación microscópica específica de los ejemplares encontrados se realizó en base a los criterios morfométricos descritos por Gupta y Kalia (1978) y Naem (2007a,b), siendo identificados como Thelazia rhodesi. Se trata de un nematodo con tropismo ocular que puede aparecer en el toro de lidia, ganaderías explotadas en régimen extensivo en zonas templadas o cálidas, cuyos condicionantes ambientales van a favorecer la proliferación de los hospedadores intermediarios y el desarrollo de la parasitosis. Por ello, la thelaziosis ha de considerarse en el diagnóstico diferencial de patologías oculares del toro de lidia cuyo cuadro sintomático incluya intranquilidad, agudeza visual disminuida, epífora y prurito en la zona periocular. En el conocimiento de los autores, esta es la primera vez que se publica un caso de parasitación por Thelazia rhodesi en el bovino de lidia.

Palabras clave: Thelazia, nematodo, toro de lidia, ocular.

\section{ABSTRACT}

Bovine thelaziosis is caused by Thelazia rhodesi, T. skrjabini and T. gulosa, although the most prevalent species in Europe is the first of them. Thelazia rhodesi is a nematode (order Spirurida, family Thelaziidae) parasitizing the eye of cattle, buffalo, zebu, and bison and, less frequently, horses and small ruminants. It is located on the corneal surface, under the lids and nictitating membrane and in the conjunctival sac. Thelazia spp. has an indirect life cycle, acting diptera of the family Muscidae as intermediate hosts. This study presents a case of thelaziosis in a fighting bull (Bos taurus), dealt in the bullring of Murcia in September 2014, coming from a farmer located in the Southwest of the Iberian Peninsula. During the first and second veterinary examinations of the animal, abundant tearing was observed, but despite this, the animal reacted positively to visual stimuli made in the visual field of both eyes, jointly and independently. The fighting bull was considered useful during the mentioned veterinary examinations, although it presented constant epiphora. During the fight, no abnormality was observed in the behavior of the bull in relation to the sense of sight. The post mortem ocular inspection in the pit of the square revealed the presence of nematodes 8-20 mm length in both the conjunctival sac and the surface of the cornea, which were extracted using tweezers and preserved in $70 \%$ ethanol. The specimens were identified as T. rhodesi according to the morphometric criteria described by Gupta and Kalia (1978) and Naem $(2007 \mathrm{a}, \mathrm{b})$. This is a nematode species with ocular tropism that can appear in the fighting bull, farms exploited in extensive regime in temperate or warm areas, whose environmental conditions will favor the proliferation of intermediate hosts and the development of parasitosis. Therefore, thelaziosis must be considered in the differential diagnosis of ocular pathologies of fighting bull whose signs include restlessness, decreased visual acuity, epiphora and pruritus in the periocular area. To the knowledge of the authors, no case of Thelazia rhodesi infection has been reported in fighting bull.

Key word: Thelazia, nematode, fighting bull, ocular.

\section{INTRODUCCIÓN}

La thelaziosis bovina está causada por Thelazia rhodesi, Thelazia skrjabini y Thelazia gulosa, aunque la especie habitual en Europa es la primera de ellas (Anderson, 2000). Thelazia rhodesi es un nematodo (orden Spirurida, familia Thelaziidae) que parasita el globo ocular del ganado vacuno, búfalos, cebúes, bisontes y, con menor frecuencia, caballos y pequeños rumiantes (Smell, 1968, Anderson,
2000; Munang'andu et al., 2011). Es un parásito de distribución mundial, pero sobre todo afecta a ganaderías explotadas en régimen extensivo en zonas templadas o cálidas, pues son condicionantes ambientales que van a favorecer la proliferación de los hospedadores intermediarios.

Se trata de un nematodo que se puede localizar en la superficie corneal, sobre la membrana nictitante, conducto naso-lacrimal, saco 
conjuntival y conductos excretores de las glándulas lacrimales (Otranto y Traversa, 2005). El parásito ha desarrollado mecanismos que le protegen frente a la acción de los factores de defensa de la lágrima, como son las lisozimas e inmunoglobulinas (Gelatt, 2007).

Thelazia spp. tiene un ciclo biológico indirecto. Las especies que afectan al ganado bovino presentan como hospedadores intermediarios dípteros de la familia Muscidae, sobre todo Musca autumnalis, que son los causantes de la transmisión de las larvas III infectivas del nematodo (Giangaspero et al., 2000, 2004; Otranto y Traversa, 2005). Los nematodos adultos se reproducen en el globo ocular del hospedador definitivo. Las hembras son vivíparas, de manera que depositan larvas I en las secreciones lagrimales del hospedador, y así serán posteriormente ingeridas por los dípteros cuando se alimenten de dichas secreciones oculares, lágrimas y conjuntiva del bóvido parasitado (Otranto y Traversa, 2004). En el organismo del díptero las larvas sufren dos mudas en 2-3 semanas, evolucionando hasta el estadio de larva III, que es la fase infectiva. Dicha larva III migra hacia la probóscide de la mosca, para ser depositada en el ojo de un nuevo hospedador cuando va a alimentarse de las secreciones oculares de este (Otranto y Traversa, 2005). Es por esto que la propagación del parásito tiene carácter estacional, principalmente durante el periodo estival, que es cuando hay mayor abundancia de los vectores que lo transmiten.

Se trata de nematodos de aproximadamente 8-20 mm de longitud y 300-500 micras de anchura, siendo más grandes las hembras que los machos. Son filiformes, con extremos afilados y de color blanco lechoso. En los ejemplares hembra, la vulva se sitúa en el extremo anterior del cuerpo, en concreto cerca de la región esofágica. El útero se caracteriza por estar repleto de huevos larvados. Posee una cutícula con estriaciones transversales profundas en ambos sexos, muy bien marcadas en el extremo anterior del cuerpo, lo que le permite fijarse y desplazarse sobre la superficie corneal del hospedador (Naem, 2007a).

La existencia de nematodos del género Thelazia, tanto en sus formas larvarias como adultas, ocasionan la aparición de sintomatología clínica/subclínica por la erosión en conjuntiva y epitelio corneal (Deepthi y Yalavarthi, 2012). Se produce una hipersecreción ocular o lagrimeo, lo que estimula la aproximación de moscas que se infectarán con las larvas I al alimentarse de las secreciones oculares del hospedador definitivo. En ocasiones, la conjuntivitis cursa con un intenso prurito que provoca que los animales parasitados se froten y, en consecuencia, se produzcan autolesiones que agravan el problema (Dacal et al., 2011). Otros signos que pueden aparecer, además del lagrimeo y la conjuntivitis, son el edema palpebral, exudados purulentos oculares e hipersensibilidad a la luz (Miller y Campbell, 1992; Van Aken et al., 1996; Dacal et al., 2011). En procesos crónicos, se producen daños en córnea apareciendo queratitis y ulceraciones que pueden dar lugar a ceguera, además de la aparición de infecciones bacterianas secundarias como las causadas por Moraxella bovis (Otranto y Travesa, 2004). La intensidad de la infección y por tanto el cuadro lesional va a depender de factores como la larga vida de Thelazia, falta de respuesta inmunológica y las reinfecciones a lo largo de la vida del hospedador (Otranto et al., 2003; Otranto y Travesa, 2004).

El objetivo del presente estudio es describir el primer caso de thelaziosis en toro de lidia (Bos taurus) originada por Thelazia rhodesi en una ganadería de la Península Ibérica y que fue lidiado en una plaza de toros.

\section{MATERIAL Y MÉTODOS}

\section{1) Animales del estudio}

El presente estudio se realizó en 30 animales de la raza bovina de lidia (Bos taurus) lidia- 
dos en la plaza de toros de la ciudad de Murcia (SE España) en la feria taurina de septiembre de 2014. Los animales pertenecían a ganaderías de la asociación ganadera Unión de Criadores del Toro de Lidia. Durante el primer y segundo reconocimiento veterinario de los animales que se llevó a cabo en la plaza de toros, se apreció que uno de los animales que iba a ser lidiado presentaba un lagrimeo abundante en ambos ojos. El toro presentaba un peso de $550 \mathrm{~kg}, 56$ meses de edad y el encaste de procedencia era Juan Pedro Domecq (Real Decreto 60/2001). La ganadería de origen está localizada en el suroeste de la Península Ibérica (Región del Alentejo, Portugal).

\section{2) Toma de muestras}

Durante la inspección post mortem realizada en el desolladero de la plaza de toros de Murcia, se procedió a la recogida de muestras del animal. En concreto, se examinaron minuciosamente los ojos, y se detectó la presencia de nematodos de aspecto blanquecino (6 en el ojo izquierdo y 5 en el ojo derecho). Los parásitos fueron extraídos con cuidado mediante unas pinzas de disección, y se conservaron en etanol al $70 \%$ para su posterior estudio en el laboratorio del área de Parasitología y Enfermedades Parasitarias del Departamento de Sanidad Animal de la Facultad de Veterinaria de la Universidad de Murcia. Además, se realizó el estudio histopatológico de un nematodo que fue conservado en formol al $10 \%$.

\section{3) Identificación del parásito}

Una vez en el laboratorio de Parasitología, los nematodos fueron extraídos, lavados en agua destilada y, finalmente, montados en preparaciones semipermanentes de lactofenol con el fin de ser aclarados. Posteriormente se procedió a su identificación específica microscópica en base a los criterios morfométricos descritos por Gupta y Kalia (1978) y Naem (2007a, b).

\section{4) Estudio histopatológico del parásito}

En el laboratorio de Histopatología el parásito fue fijado en formol. Igualmente se extrajeron los ojos y fueron fijados en formol. Posteriormente, los parásitos y diferentes partes de córnea fueron incluidos en parafina. Se realizaron cortes de $5 \mu \mathrm{m}$ de grosor que fueron teñidos en Hematoxilina-eosina (H-E) para su visualización en el microscopio óptico.

\section{RESULTADOS}

Se observó durante el primer y segundo reconocimiento veterinario en la plaza de toros de Murcia en septiembre de 2014 la existencia de un toro de lidia con lagrimeo abundante en ambos ojos. A pesar de las molestias, el animal reaccionaba positivamente a estímulos visuales realizados sobre ambos ojos, conjunta e independientemente. Así, fue dado como útil durante los citados reconocimientos veterinarios. Durante la lidia, no se observó anormalidad alguna en su comportamiento en relación con el sentido de la vista.

En la inspección post mortem, los nematodos aparecían en ambos globos oculares del toro de lidia estudiado sobre la superficie corneal, saco lagrimal y bajo la membrana nictitante (Fig. 1a y b). Se observó una leve conjuntivitis en ambos ojos. No se detectaron alteraciones macroscópicas ni microscópicas en la superficie de la córnea de los animales estudiados.

La identificación morfométrica de los nematodos demostró que eran ejemplares de Thelazia rhodesi. Se identificaron tanto machos como hembras. Se caracterizaron por tener tamaño variable entre 8 y $20 \mathrm{~mm}$ de longitud y 300500 micras de anchura, por ser filiformes, con extremos afilados y de color blanco lechoso. Se observó que presentaban una cutícula con estriaciones transversales profundas, muy bien marcadas en el extremo anterior del cuerpo, lo que les permite fijarse y desplazarse sobre la superficie corneal del hospedador (Fig. 2a). La 


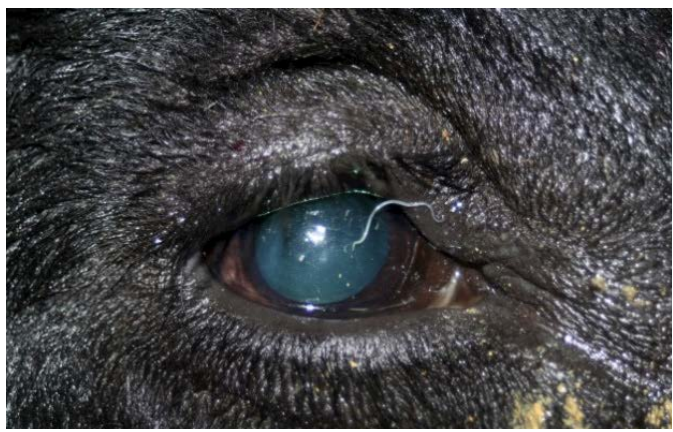

Figura 1a. Toro de lidia en la inspección post mortem con nematodos en la superficie corneal y la zona palpebral.

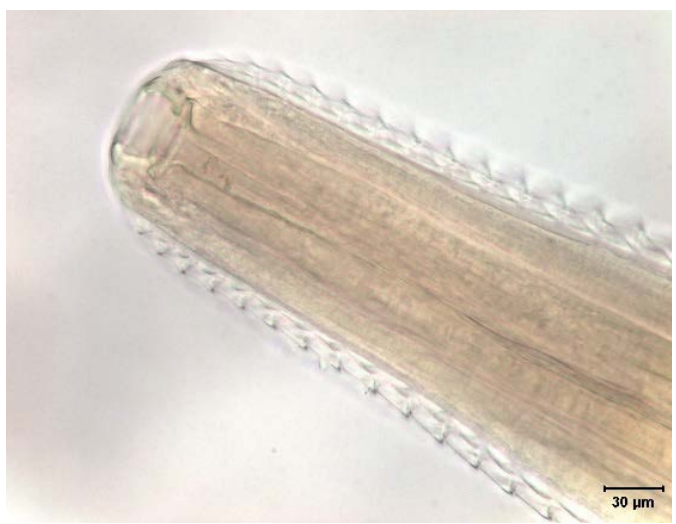

Fig. 2a. Adulto de T. rhodesi. A, extremo anterior con cápsula bucal (x40).

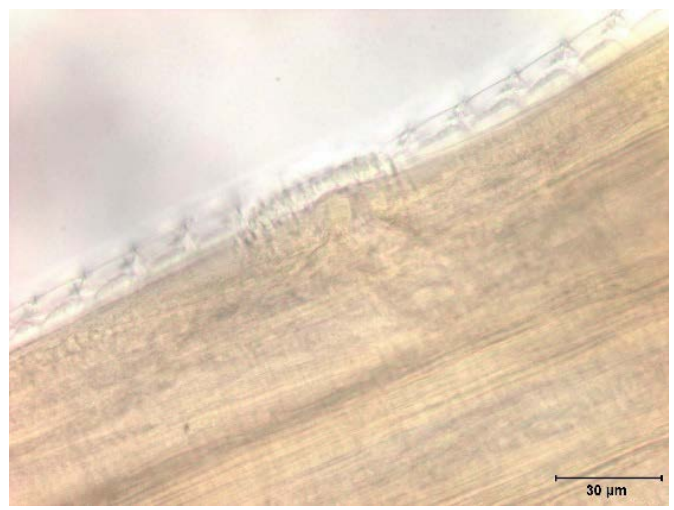

Fig. 3a. Hembra de Thelazia rhodesi. Detalle de la zona vulvar (x63).

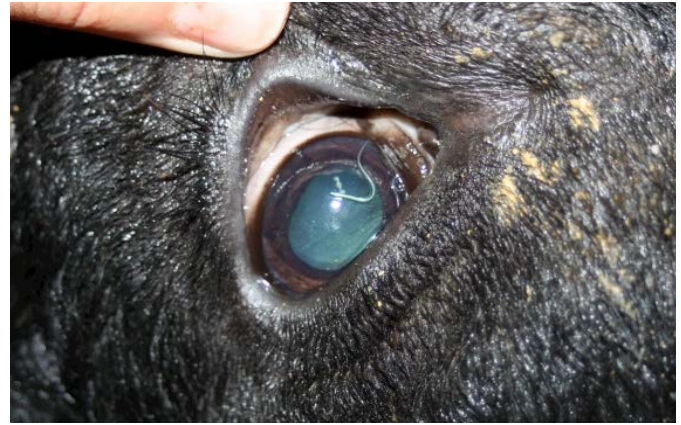

Figura 1b. Toro de lidia en la inspección post mortem con nematodos en superficie corneal y sobre la membrana nictitante.

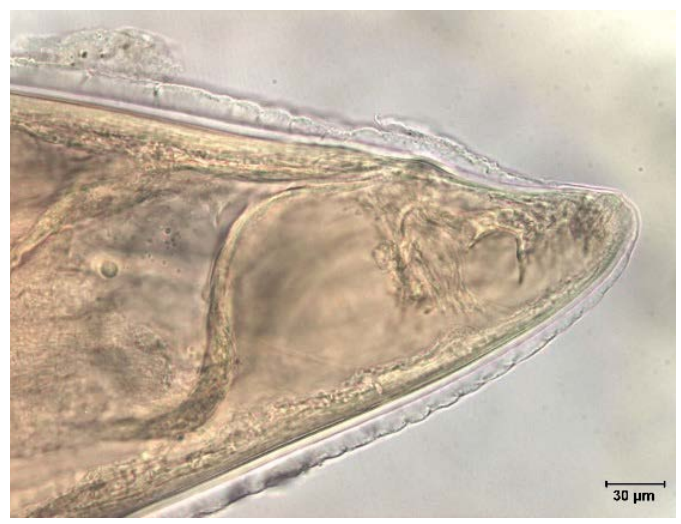

Fig. 2b. Adulto de T. rhodesi. Porción distal de una hembra (40x).

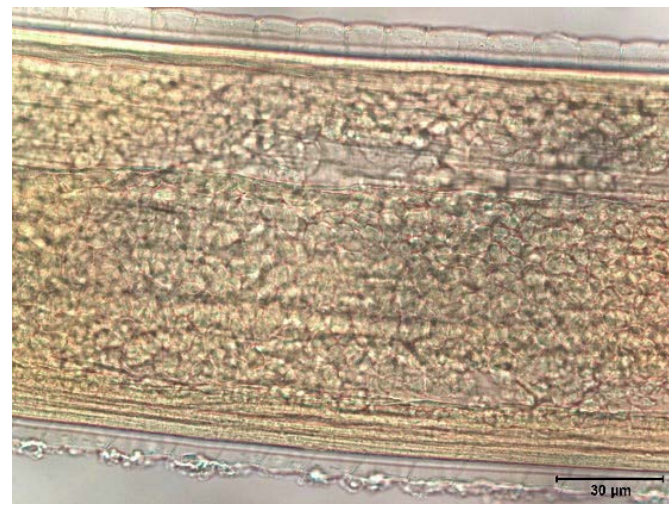

Fig. 3b. Hembra de Thelazia rhodesi. Presencia de huevos en la zona próxima a la vulva (x63). 


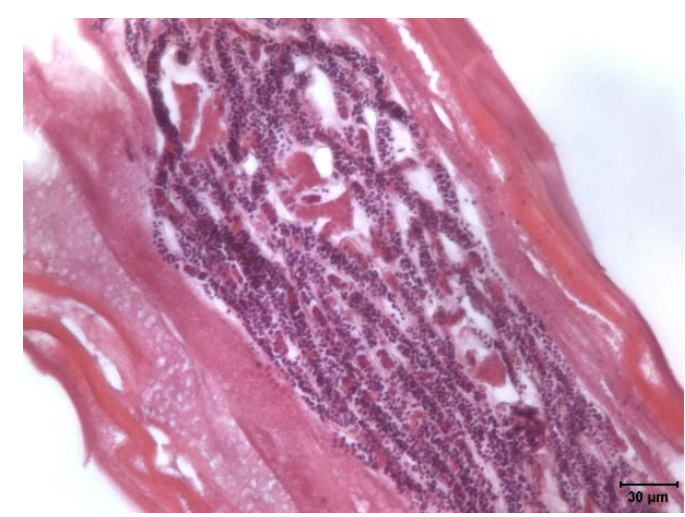

Fig. 4a. Hembra de Thelazia rhodesi. Útero con huevos y abundantes larvas (H-E x 40).

porción final aparecía acuminada en la zona del ano y las estriaciones más suaves (Fig. 2b).

En los ejemplares hembra, la vulva se situaba en el tercio anterior del cuerpo, en concreto cerca de la región esofágica (Fig. 3a). El útero se caracterizó por ocupar la parte central del parásito y en hembras grávidas estaba repleto de huevos y larvas. Mientras que con el estudio parasitológico del animal se pudieron diferenciar bien los huevos (Fig. 3b), mediante el estudio histopatológico se pudieron diferenciar en el interior del útero tanto la presencia de huevos embrionados como de larvas (Fig. 4 a y b).

\section{DISCUSIÓN}

En este caso clínico describimos la presencia de adultos de Thelazia rhodesi en los ojos de un toro de lidia en el que, durante el reconocimiento veterinario, se detectó una abundante epífora, y que fue lidiado con posterioridad sin alteraciones aparentes en la visión en la plaza de toros de Murcia.

Thelazia spp. es un nematodo espirúrido de distribución mundial, aunque en Europa hay muy pocos estudios epidemiológicos acerca de la prevalencia de este parásito en bovinos. La thelaziosis bovina ha sido descrita en Ita-

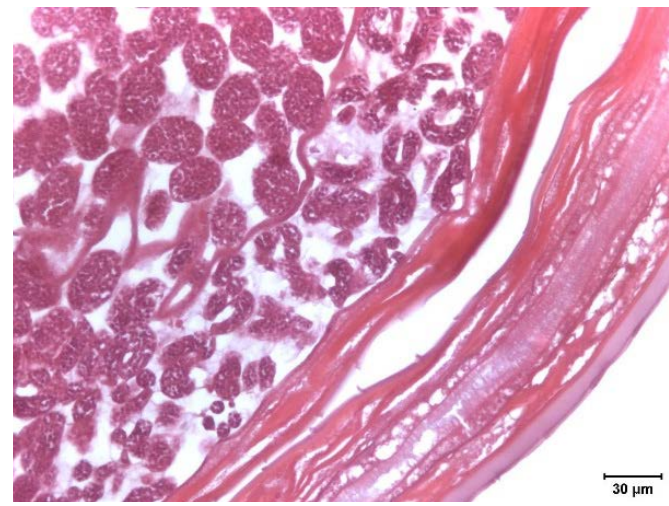

Fig. 4b. Hembra de Thelazia rhodesi. Detalle de huevos embrionados y larvas en útero (H-E $\mathrm{x} 40$ ).

lia y Gran Bretaña (Giangaspero et al., 2000; Tweedle et al., 2005), con unas prevalencias del 7,2\% y 1,5\%, respectivamente. En España, T. rhodesi fue descrito por primera vez por Jordano (1945). La prevalencia de la thelaziosis es mayor en zonas en donde los animales están expuestos durante mayor tiempo a un posible contacto con las moscas vectores (Otranto y Traversa, 2005). Así afecta fundamentalmente a ganaderías explotadas en régimen extensivo en zonas templadas o cálidas, pues son condicionantes ambientales que van a favorecer la proliferación de las moscas que actúan como hospedadores intermediarios. Sin embargo, a pesar de su amplia distribución, son muy escasos los estudios publicados sobre este parásito en España. Considerando que la raza de lidia se asocia tradicionalmente a sistemas de explotación en régimen extensivo (Sánchez Belda, 1984) en la Península Ibérica y Sudamérica, es posiblemente una raza propensa al desarrollo de esta parasitosis, aunque hasta el presente estudio no se haya descrito ningún caso. En este sentido consideramos que se trata de una parasitosis cuya prevalencia está infraestimada por la propia idiosincrasia de las explotaciones de ganado de lidia. En concreto, la dificultad en el manejo del toro de lidia hace que sea muy 
complicado observar patologías oculares, salvo que ocasionen pérdida de la función visual, que puedan ser diagnosticadas a nivel de campo o en un reconocimiento veterinario previo a la lidia. Además, las inspecciones post mortem se celebran en unas condiciones excepcionales tras los festejos en los desolladeros de las plazas de toros y van encaminadas a la aptitud de la canal para consumo humano, no siendo los ojos evaluados de forma rutinaria y concienzuda. El hecho de que los festejos taurinos de la Feria de Murcia se celebren en el mes de septiembre, fecha en la que encontramos este caso, es sin duda favorecedor para observar casos de thelaziosis, puesto que es una nematodosis con cierto grado de estacionalidad (Arbuckle y Khalil, 1978); en concreto, durante el verano y el comienzo del otoño es cuando es más probable que los animales se vean parasitados, dado que la abundancia de las moscas (vectores intermediarios) en dicho periodo favorece la transmisión del parásito $\mathrm{y}$, en consecuencia, la presencia de nematodos adultos (Khedri et al., 2016).

La thelaziosis suele pasar desapercibida, cursa de manera subclínica, pero puede llegar a provocar disminución de la capacidad visual de los animales parasitados, intranquilidad, descarga nasal, así como un lagrimeo continuo que atrae a más dípteros que se alimentan de secreciones orgánicas del hospedador. Además, se han descrito casos de queratoconjuntivitis, opacidad corneal, edema palpebral, quistes subconjuntivales, e incluso ceguera (Miller et al., 1992; Van Aken et al., 1996; Deepthi y Yalavarthi, 2012; Khedri et al., 2016; Hassan et al., 2017). Los casos donde aparecen alteraciones oculares más severas con epífora, queratitis o conjuntivitis, suelen relacionarse con la presencia de hembras grávidas de Thelazia, por la importancia de las larvas en la patogénesis de la enfermedad (Smeal, 1968). En el caso que describimos aparecieron hembras grávidas y, sin embargo, las alteraciones oculares encontradas fueron leves; sólo se apreció la presencia de lagrimeo continuo, lo que podría estar rela- cionado con que la parasitación estuviera en las fases iniciales $y$, en consecuencia, todavía no fuesen manifiestas las lesiones oculares debidas a la acción patógena de las larvas. En este sentido, consideramos que el lagrimeo existente estaba originado por la presencia de los nematodos adultos en la superficie corneal y membrana nictitante (Otranto y Traversa, 2005).

El estudio de las alteraciones oculares es fundamental en los reconocimientos veterinarios de este tipo de animales antes de las corridas de toros (Rodríguez, 1991), ya que el sentido de la vista debe estar en perfecto estado, a fin de evitar anomalías de comportamiento del animal debido a un defecto en la visión durante el desarrollo de la lidia. Es por ello por lo que se debe hacer un examen minucioso de los ojos y discernir entre lagrimeos de tipo puntual por algún agente irritante exógeno (como, por ejemplo, el producido por cuerpos extraños) de aquellos que sean consecuencias de procesos patológicos. En el caso de los primeros, hay ocasiones que remiten de forma espontánea, al desaparecer la causa que origina la irritación, mientras que cuando hay un proceso patológico suelen ser persistentes y la secreción lacrimal se puede alterar, pudiendo cursar con lesiones oculares y, en consecuencia, alteraciones de la visión. Las infecciones oculares en bovinos son muy diversas; así, en el toro de lidia, la queratoconjuntivitis tiene gran importancia, pues son procesos frecuentes que pueden tener un origen traumático o infeccioso por bacterias como Moraxella bovis, aunque también pueden intervenir otros agentes como Mycoplasma spp., Pseudomonas spp., Streptococcus spp., y Staphylococcus spp. (Jubb et al., 2007; Mcgavin y Zachary, 2007). Estas cursan con queratitis, conjuntivitis, lagrimeo y prurito en sus fases iniciales que hay que diferenciar de afecciones como la queratoconjuntivitis parasitaria descrita en este caso, siendo además un proceso más frecuente en animales jóvenes y épocas calurosas (Jubb et al., 2007). Por tanto, en el toro de lidia las alteraciones originadas por Thelazia 
rhodesi han de considerarse en el diagnóstico diferencial de patologías oculares cuyos signos clínicos incluyan lagrimeo y prurito.

\section{BIBLIOGRAFÍA}

Arbuckle, J.B., Khalil, L.F., 1978. A survey of Thelazia worms in the eye of the British cattle. Vet. Rec. 102: 207-10.

Anderson, R.C. 2000. Nematode Parasites of Vertebrates: Their Development and Transmission. ( $2^{\mathrm{a}}$ ed.). Oxon: CABI Publishing.

Dacal, V., Vázquez, L., Pato, F.J., Francisco, I., Cazapal-Monteiro, C., Romasanta, A., Arias, M.S., Panadero, R., Díez-Baños, P., Paz-Silva, A., Sánchez-Andrade, R. 2011. Hallazgo de infección por nematodos del género Thelazia (Boso, 1819) en un perro de Salamanca (España). Rev. Ibero-Latinoam. Parasitol: 70(1): 109-113.

Deepthi, B., Yalavarthi, Y. 2012. Eye worm infection in a Cattle. A case report. Vet. World. 5 (4), 236-237.

Gelatt, K.N. 2007. Veterinary Ophthalmology, Vol. 1. (4 ${ }^{\mathrm{o}}$ ed.). Iowa: Blackwell Publishing. Giangaspero, A., Otranto, D., Vovias, N., Puccini, V. 2000. Thelazia gulosa Railliet \& Henry, 1910 and T. skrjabini Erschow, 1928 infection in southern Europe (Italy). Parasite. 7 (4), 327-329.

Giangaspero, A., Traversa, D., Otranto, D. 2004. Ecology of Thelazia spp. in cattle and their vectors in Italy. Parassitologia 46 (1-2), 257-259.

Gupta, N.K., Kalia, D.C. 1978. On nematodes of some live-stock animals in India, Parte I. Rev. Ibér. Parasitol, 38 (1-2), 35-61.

Hassan, E.B., Moshaverinia, A., Sheedfar, F., Mccowan, C., Bazargani, T.T., Hosseinzadeh, A., Saghafi, R., Ashrafihelan, J., Beveridge, I. 2017. A report of the unusual lesions caused by Thelazia gulosa in cattle. Vet. Parasitol. Reg. Stud. Reports. 7: 62-65.

Jordano, D. 1945. Primeros casos de conjuntivitis verminosa en terneros, debidos a la "The- lazia rhodesi" (Desmarest, 1827). Zootecnia VI (11-12), 28-31.

Jubb, K.V.F., Kennedy, P.C., Palmer, N. 2007. Pathology of Domestic Animals. 5 ed. Saunders, San Diego.

Khedri, J., Radfar, M.H., Borji, H., Azizzadeh, M. 2016. Epidemiological Survey of Bovine Thelaziosis in Southeastern of Iran. Iran Journal of Parasitology, 11 (2), 221-225.

Mcgavin, M.D., Zachary J.F. 2007. Pathologic Basis of Veterinary Disease. $4^{\mathrm{a}}$ ed. Mosby, Inc. St. Louis. Misouri.

Miller, P.E., Campbell, B.G. 1992. Subconjuntival cyst associated with Thelazia gulosa in a calf. J. Am. Vet. Med. Assoc. 201 (7): 1058-1060.

Munang'andu, H.M., Chembensofu, M., Siamudaala, V.M., Munyeme, M., Matandiko, W. 2011. Thelazia rhodesii in the African Buffalo, Syncerus caffer, in Zambia. Korean J. Parasitol. 49 (1): 91-94.

Naem, S. 2007a. Morphological differentiation among three Thelazia species (Nematoda: Thelaziidae) by scanning electron microscopy. Parasitol. Res. 2007 101(1):145-51.

Naem, S. 2007b. Thelazia rhodesi (Spirurida, Thelaziidae), bovine eyeworm: morphological study by scanning electron microscopy, Parasitol Res. 100 (4): 855-860.

Otranto, D., Ferroglio, E., Lia, R. P, Traversa D., Rossi, L. 2003. Current status and epidemiological observations of Thelazia callipaeda (Spirurida, Thelaziidae) in dogs, cats and foxes in Italy: a 'coincidence' or a parasitic disease of the Old Continent? Vet. Parasitol. 116: 315-325.

Otranto, D., Traversa, D. 2004. Molecular characterization of the first internal transcribed spacer of ribosomal DNS of the most common species of eyeworms (Thelazioidea: Thelazia). J. Parasitol. 90 (1): 185-188.

Otranto, D., Traversa, D. 2005. Thelazia eyeworm: an original endo- and ecto-parasitic nematode. Trends. Parasitol. 21(1): 1-4. 
Rodríguez Montesinos, A. 1991. En: Entre campos y ruedos. Ed. Ibercaja y Consejo General de Colegios Veterinarios de España. Madrid.

REAL DECRETO 60/2001, de 26 de enero, sobre prototipo racial del toro de lidia. BOE núm. 38. 13/02/2001.

Sánchez Belda, A. 1984. Razas bovinas españolas. Ed. Ministerio de Agricultura, Pesca y Alimentación. Madrid.

Smeal, M.G. 1968. Observations on the occurrence of Thelazia or eyeworm infection of cattle in Northern New South Wales. Aust. Vet. J. 44(11): 516-521.

Tweedle, D.M., Fox, M.T., Gibbons, L.M., Tennant, K.V. 2005. Change in the prevalence of Thelazia species in bovine eyes in England. Vet. Rec. 157: 555-556.

Van Aken, D.V., Dargantes, A.P., Lagapa, J.T., Vercruysse, J. 1996. Thelazia rhodesi (Desmarest, 1828) infections in cattle in Mindanao, Philippines. Vet. Parasitol. 66: 125-129. 